\title{
Administration of plasmacytoid dendritic cell-stimulative lactic acid bacteria is effective against dengue virus infection in mice
}

\author{
HIROAKI SUZUKI ${ }^{1}$, RYOHEI TSUII ${ }^{1}$, MIHO SUGAMATA ${ }^{1}$, NAOKI YAMAMOTO ${ }^{2,3}$, \\ NORIO YAMAMOTO ${ }^{4}$ and OSAMU KANAUCHI ${ }^{1}$
}

\author{
${ }^{1}$ Research Laboratories for Health Science and Food Technologies, Kirin Company, Ltd., Yokohama-shi, Kanagawa 236-0004; \\ ${ }^{2}$ National Institute of Infectious Diseases, Tokyo 162-8640; ${ }^{3}$ Tokyo Medical and Dental University, Tokyo 113-8519; \\ ${ }^{4}$ Department of Infection Control Science, Graduate School of Medicine, Juntendo University, Tokyo 113-8421, Japan
}

Received July 20, 2018; Accepted October 17, 2018

DOI: $10.3892 / \mathrm{ijmm} .2018 .3955$

\begin{abstract}
Dengue virus (DENV), a mosquito-borne flavivirus, causes an acute febrile illness that is a major public health problem in the tropics and subtropics globally. However, methods to prevent or treat DENV infection have not been well established. It was previously demonstrated that Lactococcus lactis strain plasma (LC-plasma) has the ability to stimulate plasmacytoid dendritic cells (pDCs). As pDCs are key immune cells that control viral infection by producing large amounts of type I interferons (IFN), the present study evaluated the effect of LC-plasma on DENV infection using a mouse infectious DENV strain. Mice were divided into two groups and the test group was orally administered LC-plasma for two weeks. Two weeks following administration, the mice were infected with DENV and the relative viral titers and the expression of the inflammatory genes in DENV-infected tissue were measured using reverse transcription-quantitative polymerase chain reaction (RT-qPCR). The relative viral titers were notably lower in the DENV-infected tissues compared with the control group when LC-plasma was orally administered prior to DENV infection. Furthermore, the
\end{abstract}

Correspondence to: Mr. Hiroaki Suzuki, Research Laboratories for Health Science and Food Technologies, Kirin Company, Ltd., 1-13-5 Fukuura Kanazawa-ku, Yokohama-shi, Kanagawa 236-0004, Japan

E-mail: hiroaki_suzuki@kirin.co.jp

Abbreviations: DENV, dengue virus; IFNs, interferons; ISGs, IFN-stimulated genes; LAB, lactic acid bacteria; LC-plasma, Lactococcus lactis strain plasma; pDCs, plasmacytoid dendritic cells; cDCs, conventional dentritic cells; TLR, toll-like receptors; ODN, oligodeoxyribonucleotides; MyD88, myeloid differentiation primary response 88 ; BMDC, bone-marrow derived dendritic cells

Key words: dengue virus, lactic acid bacteria, plasmacytoid dendritic cells, type I interferons, anti-viral factors expression of the inflammatory genes associated with DENV infection was also reduced by LC-plasma administration. To investigate how LC-plasma administration controls DENV infection, the present study examined anti-viral gene expression, which is critical for the viral clearance induced by type I IFN. Two weeks subsequent to the administration of LC-plasma, the expression of anti-viral gene was measured using RT-qPCR. Oral intake of LC-plasma enhanced anti-viral gene expression in DENV-infected spleen tissue. To clarify the detailed mechanism, in vitro co-culture studies using bone-marrow derived DC (BMDC) were performed. BMDC were stimulated with LC-plasma in combination with anti-IFN- $\alpha / \beta$ antibody and the expression of anti-viral genes was measured. In vitro studies revealed that the effect of LC-plasma on anti-viral genes was dependent on type I IFN. Based on these results, LC-plasma may be effective against DENV infection by stimulating pDCs, which results in the increased production of anti-viral factors.

\section{Introduction}

Dengue viruses (DENV) are single-stranded RNA viruses in the genus Flavivirus (1). They are one of the most widespread mosquito-borne viruses and are categorized into four serotypes (DENV 1-4) (2). DENV infection results in dengue fever and its more severe forms, dengue hemorrhagic fever and dengue shock syndrome $(3,4)$. Dengue hemorrhagic fever and dengue shock syndrome are characterized by increased vascular permeability, plasma leakage into tissues, thrombocytopenia and hemorrhaging within internal organs, which are thought to be immune-mediated diseases (5). According to the World Health Organization, 50-100 million individuals are estimated to develop dengue fever annually in tropical and subtropical regions of the world such as South-East Asian and South American countries, and 20,000 cases of mortality from dengue fever are estimated to occur each year (6,7). At present, however, there are few preventative antiviral drugs or vaccines against DENV infection. Therefore, effective therapeutics and/or prophylaxis against DENV infection are urgently required (8). 
Type I interferons (IFNs) are cytokines that enhance immunity to viruses or intracellular pathogens $(9,10)$. By signaling through specific receptors, type I IFNs activate a janus kinase/signal transducer and activator of transcription-dependent signaling cascade that increases the expression of hundreds of IFN-stimulated genes (ISGs) to induce the antiviral state (11). A number of reports have demonstrated that DENV is sensitive to type I IFNs, and that type I and II IFN-receptor-deficient mice are highly susceptible to DENV infection (12-15). In addition, a number of IFN-inducible anti-viral genes have been reported to inhibit DENV infection (16-18).

Plasmacytoid dendritic cells (pDCs) represent a unique immune cell type that is characterized by the production of large quantities of type I IFNs in response to viruses or self-nucleic acids $(19,20)$. pDCs express Toll-like receptor (TLR) 7 and 9 within their endosomes. TLR7 recognizes RNA viruses, endogenous RNA and synthetic oligoribonucleotides, and TLR9 senses non-methylated CpG DNA derived from viruses and bacteria, endogenous DNA and synthetic $\mathrm{CpG}$ oligodeoxyribonucleotides (ODN) (21-23). Recognition of these nucleic acids by TLR7 or TLR9 in pDCs results in the production of type I IFNs through the myeloid differentiation primary response gene 88 (MyD88)-IFN regulatory factor 7 pathway (24).

Lactic acid bacteria (LAB) have been reported to have immune-modulatory effects on hosts through the activation of innate immune cells, including macrophages or conventional DCs (cDCs) (25). Although a number of pathogenic bacteria have been demonstrated to stimulate pDCs (26), beneficial bacteria, including LAB, have been less studied in terms of activating pDCs (27). It was previously reported that Lactococcus lactis strain plasma (LC-plasma, also known Lactococcus lactis subsp. lactis JCM 5805) stimulates murine pDCs to produce large quantities of type I IFNs in association with cDCs (28). It was further demonstrated that the effect of LC-plasma on the activation of pDCs is dependent on the TLR9-MyD88 signaling pathway. As pDCs serve a pivotal function in anti-viral immune responses through type I IFN production, the effect of LC-plasma on virus infection was verified using virus infection mouse models. It was demonstrated that murine para-influenza virus-infected mice that were fed LC-plasma demonstrated a markedly improved survival rate compared with controls (29). Furthermore, the oral administration of LC-plasma protected mice against rotavirus infection (30). Therefore, the activation of $\mathrm{pDCs}$ by LAB administration appeared to defend the host against viral infection.

In the present study, the effect of a pDC-stimulating LAB, LC-plasma, on DENV infection in mice was examined. To verify the effectiveness of LC-plasma against DENV infection, the virus titer and the expression of inflammatory genes in DENV-infected tissues when LC-plasma was orally administered were examined. In addition, to elucidate the mechanism of protection against DENV infection by LC-plasma administration, the present study focused on anti-viral gene expression, which is assumed to be effective for preventing DENV infection (31). Finally, an in vitro study was performed and examined the influence of type I IFNs induced by LC-plasma on the enhancement of anti-viral gene expression. To date, whereas certain types of LAB have been reported to have immune-modulatory effects on host immunity, it has remained unclear whether LAB exerts an effect on DENV infection, which is a major threat to human health. This is the first report, to the best of our knowledge, to investigate whether the administration of LAB is effective against DENV infection in vivo.

\section{Materials and methods}

Mice. A total of 56 C57BL/6J female mice (6 week-old; weight, 15-25 g) were purchased from Charles River Laboratories Japan, Inc. (Kanagawa, Japan). All animals were maintained in a specific pathogen-free facility under a $12 \mathrm{~h}$ light/dark cycle with ad libitum access to water and a basic diet of AIN93G pellets (Oriental Yeast Co., Ltd., Tokyo, Japan). The temperature in the facility was maintained at $22-25^{\circ} \mathrm{C}$ and $40-60 \%$ humidity. All animals were sacrificed using $5 \%$ isoflurane exposure until 2-3 min after the breathing had ceased. All animal experiments were performed in accordance with the guidelines for the care and use of laboratory animals of Juntendo University (Tokyo, Japan) and Kirin Company, Ltd. (Kanagawa, Japan). The study was ethically approved by the Committee for Animal Experimentation of Juntendo University and Kirin Company, Ltd. All efforts were made to minimize animal suffering.

$L A B$ strains. LAB strains tested in this study were purchased from collections held at the Japan Collection of Microorganisms (Tsukuba, Japan) and American Type Culture Collection (ATCC; Manassas, VA, USA). Lactobacillus rhamnosus strain GG (53103; ATCC) was grown at $37^{\circ} \mathrm{C}$ for $48 \mathrm{~h}$ in MRS broth (Oxoid; Thermo Fisher Scientific, Inc., Waltham, MA, USA) and LC-Plasma was grown at $30^{\circ} \mathrm{C}$ for $48 \mathrm{~h}$ in M17 broth (Oxoid; Thermo Fisher Scientific, Inc.) according to the manufacturer's protocol. Cultured LAB strains were washed twice with sterile distilled water, heat-killed at $100^{\circ} \mathrm{C}$, lyophilized and suspended in phosphate-buffered saline for the in vitro study.

Oral intake of LC-plasma. Six week-old C57BL/6J female mice were acclimatized for 1 week with ad libitum access to water and a basic diet of AIN93G pellets. Control groups were fed with $4 \mathrm{~g} /$ day AIN93G pellets and test groups were fed with $4 \mathrm{~g}$ /day AIN93G pellets containing $1 \mathrm{mg}$ heat-killed LC-plasma during the course of the study.

Virus infection study. A total of 32 mice were divided into two groups (control; n=16, LC-plasma; $n=16$ ) and housed with 4 animals/cage following LC-plasma oral administration for 2 weeks as described above. A $5 \times 10^{7} \mathrm{pfu} / \mathrm{ml}$ DENV virus solution was prepared, and mice were intraperitoneally injected with $200 \mu \mathrm{l}$ solution. Mice were monitored every day subsequent to viral infection by monitoring the intake of food and water, the state of breathing and other abnormal behaviors. As the virus titer peaks at 2-3 days following virus infection, mice were sacrificed 2-3 days subsequent to infection, and spleens, liver and blood were harvested. The spleen and liver were homogenized in TRIzol reagent (Life Technologies; Thermo Fisher Scientific, Inc.) for $1 \mathrm{~min}$ at room temperature followed by RNA extraction. Total RNA was extracted by phenol/chloro- 
form/isoamyl alcohol extraction method. Blood samples were collected in EDTA-treated tubes (Terumo Co., Tokyo, Japan) that were subjected to centrifugation at $400 \mathrm{x}$ g for $20 \mathrm{~min}$ at room temperature. Subsequent to centrifugation, plasma was obtained from blood samples for NS1 enzyme-linked immunosorbent assay (ELISA).

Non-virus infection study. A total of 20 mice were divided into two groups (control, $n=10 ;$ LC-plasma, $n=10$ ) and housed at 1/cage following LC-plasma oral administration for 2 weeks as described above. After 2 weeks of oral intake of LC-plasma, the mice were sacrificed and the spleens were collected. The spleen was minced in $\mathrm{Mg}^{2+}$ - and $\mathrm{Ca}^{2+}$-free Hank's Balanced Salt solution (Gibco; Thermo Fisher Scientific, Inc.) and digested with $1 \mathrm{mg} / \mathrm{ml}$ collagenase (Sigma-Aldrich; Merck KGaA, Darmstadt, Germany) for $20 \mathrm{~min}$ at $37^{\circ} \mathrm{C}$. EDTA (Invitrogen; Thermo Fisher Scientific, Inc.) was adjusted to $30 \mathrm{mM}$ and the sample was incubated for $10 \mathrm{~min}$ at room temperature. Tissue lysates were filtered through a $100 \mu \mathrm{m}$ nylon cell strainer and erythrocytes were removed by exposure to $2 \mathrm{ml}$ of Red Blood Cell Lysis Buffer (Sigma-Aldrich; Merck KGaA) for 5 min at room temperature. Tissue lysates were filtered through a $70 \mu \mathrm{m}$ nylon cell strainer again and filtered tissue lysates were used for splenocytes. CD11 $\mathrm{c}^{+}$cells were separated from splenocytes using CD11c MicroBeads (Miltenyi Biotec, Inc., Cambridge, MA, USA). The mediated splencytes and CD11c ${ }^{+}$cells were suspended in RNAlater Stabilization solution (Thermo Fisher Scientific, Inc.).

Viral strains and growth conditions. DENV strain Eden2 was used in the present study. DENV Eden2 was provided by Dr. Ashley L. St. John of Duke-NUS Medical School (College Road, Singapore) and C6/36 cells and Baby hamster kidney 21 cells were provided by Dr. Yo-ichi Suzuki of Osaka Medical College (Osaka, Japan). DENV Eden2 was grown in Aedes albopictus C6/36 cells. C6/36 cells were seeded in plastic flasks and grown in RPMI-1640 medium (Thermo Fisher Scientific, Inc.) supplemented with $10 \%$ heat-inactivated fetal bovine serum (Moregate Biotech, Bulimba, Australia) and $100 \mathrm{U} / \mathrm{ml}$ penicillin-streptomycin (Thermo Fisher Scientific, Inc.). Once the cells were fully confluent, DENV Eden2 was transferred to $\mathrm{C} 6 / 36$ flasks at a multiplicity of infection of 0.01 . Viral samples were incubated for $>1 \mathrm{~h}$ at $28^{\circ} \mathrm{C}$, and subsequently the medium was substituted using RPMI-1640 medium supplemented with $2 \%$ inactivated fetal bovine serum. Cells were cultured for 5-7 days, and culture supernatants were collected. Cell debris was removed by centrifugation at $800 \mathrm{x} \mathrm{g}$ for $10 \mathrm{~min}$ at $4^{\circ} \mathrm{C}$, and supernatants containing the virus were stored at $-80^{\circ} \mathrm{C}$. Viral titers were determined by plaque assays using Baby hamster kidney 21 cells. Cells were seeded in 24-well plates in RPMI-1640 medium supplemented with $10 \%$ inactivated fetal bovine serum and $100 \mathrm{U} / \mathrm{ml}$ penicillin-streptomycin. Cells were maintained in a humidified incubator at $5 \% \mathrm{CO}_{2}$ and $37^{\circ} \mathrm{C}$ until they reached confluence. Then, the medium was removed and the monolayer was infected with $200 \mathrm{ml}$ of $10-, 10^{2}-, 10^{3}-, 10^{4}-, 10^{5}$ - and $10^{6}$-fold diluted virus samples. The plates were incubated for $1 \mathrm{~h}$ at $37^{\circ} \mathrm{C}$. After $1 \mathrm{~h}$, cells were washed and then $0.5 \mathrm{ml} 1 \%$ carboximethylcellulose (Sigma-Aldrich; Merck KGaA) mixed with RPMI-1640 medium supplemented with $2 \%$ inactivated fetal bovine serum and $100 \mathrm{U} / \mathrm{ml}$ penicillin-streptomycin was added to each well. The plates were maintained in a humidified incubator at $37^{\circ} \mathrm{C}$ and $5 \% \mathrm{CO}_{2}$ for 5-6 days. Subsequent to this period, the cells were fixed with $4 \%$ paraformaldehyde phosphate buffer solution (Wako Pure Chemical Industries, Ltd., Osaka, Japan) at room temperature for $1 \mathrm{~h}$ and the carboxymethylcellulose plugs were removed by washing with water. Plaques were visualized by staining with a solution of $1 \%$ crystal violet (Wako Pure Chemical Industries, Ltd.) in 20\% ethanol for 2-3 min at room temperature.

NS1 ELISA. Concentrations of NS1 antigen in plasma samples were measured using a commercially available DENV NS1 ELISA kit purchased from Biocompare (South San Francisco, CA, USA).

In vitro co-culture assays. BM cells were isolated from $\mathrm{C} 57 \mathrm{BL} / 6 \mathrm{~J}$ mice, and erythrocytes were removed by exposure to $1 \mathrm{ml}$ of Red Blood Cell Lysis Buffer (Sigma-Aldrich; Merck KGaA) for $1 \mathrm{~min}$ at room temperature. Cells were cultured at a density of $5 \times 10^{5}$ cells $/ \mathrm{ml}$ for 7 days in RPMI-1640 medium supplemented with $1 \mathrm{mM}$ sodium pyruvate (Life Technologies; Thermo Fisher Scientific, Inc.), $2.5 \mathrm{mM}$ HEPES (Life Technologies; Thermo Fisher Scientific, Inc.), 100 U/ml penicillin-streptomycin (Life Technologies; Thermo Fisher Scientific, Inc.), $50 \mu \mathrm{M}$ 2-mercaptoethanol (Life Technologies; Thermo Fisher Scientific, Inc.), $10 \%$ fetal bovine serum and $100 \mathrm{ng} / \mathrm{ml}$ FMS-like tyrosine kinase 3 ligand (Flt-3L; R\&D Systems, Inc., Minneapolis, MN, USA) at $37^{\circ} \mathrm{C}$ in a humidified atmosphere of $5 \% \mathrm{CO}_{2}$ and $95 \%$ air. After 7 days of culturing, cells were washed with RPMI-1640 medium and then incubated at a density of $2 \times 10^{5}$ cells $/ \mathrm{ml}$ with $1 \mu \mathrm{M} \mathrm{CpG}$ ODN (InvivoGen, Toulouse, France) or $10 \mu \mathrm{g} / \mathrm{ml} \mathrm{LC}$-plasma or Lactobacillus rhamnosus strain GG (ATCC 53103) at $37^{\circ} \mathrm{C}$ for $24 \mathrm{~h}$. To block TLR9 signals, a TLR9 antagonist (InvivoGen) was added with LC-plasma. To neutralize IFN- $\alpha / \beta$, anti-IFN- $\alpha$ and anti-IFN- $\beta$ antibody (PBL Assay Science, Piscataway, NJ, USA) was added with LC-plasma. Subsequent to culturing, the supernatant was collected and other BMDC or splenocytes were cultured with the collected supernatant for $24 \mathrm{~h}$. Subsequent to culturing with the collected supernatant, each cell sample was collected for RNA extraction.

Reverse transcription-quantitative polymerase chain reaction (RT-qPCR). Total RNA from spleen and liver was extracted using TRIzol reagent (Ambion; Thermo Fisher Scientific, Inc.). Subsequent to the extraction of tissue total RNA, a purification step was performed using an RNeasy mini kit (Qiagen $\mathrm{GmbH}$, Hilden, Germany) according to the manufacturer's protocol. Total RNA from splenocytes, CD11 ${ }^{+}$cells or BMDC was extracted using an RNeasy mini kit. To synthesize complementary DNA, purified total RNA samples were reverse transcribed using an iScript cDNA synthesis kit (Bio-Rad Laboratories, Inc., Hercules, CA, USA) according to the manufacturer's protocol. RT-qPCR (qRT-PCR) was performed using SYBR Premix Ex Taq (Takara Bio, Inc, Otsu, Japan) and a LightCycler 480 (Roche Diagnostics, Basel, Switzerland). The PCR conditions were as follows: 2 -Step Cycling, $95^{\circ} \mathrm{C}$ for $10 \mathrm{sec}$ hold, 45 cycles of $95^{\circ} \mathrm{C}$ for $5 \mathrm{sec}$ and $60^{\circ} \mathrm{C}$ for $20 \mathrm{sec}$. All values were normalized to the expression of GAPDH 
and calculated using the $2^{-\Delta \Delta \mathrm{Cq}}$ method (32). Specific forward and reverse primer pairs that were used are listed as follows: GAPDH forward, 5'-TGTGTCCGTCGTGGATCTGA-3' and reverse, 5'-TTGCTGTTGAAGTCGCAGGAG-3'; interleukin (IL)-6 forward, 5'-CCACTTCACAAGTCGGAGGCT TA-3' and reverse, 5'-TGCAAGTGCATCATCGTTGTTC-3'; tumor necrosis factor $\alpha(\mathrm{TNF} \alpha)$ forward, 5'-ACTCCAGGC GGTGCCTATGT-3' and reverse, 5'-GTGAGGGTCTGGGCC ATAGAA-3'; IL-1 $\beta$ forward, 5'-TCCAGGATGAGGACATGA GCAC-3' and reverse, 5'-GAACGTCACACACCAGCAGGT TA-3'; monocyte chemoattractant protein 1 (MCP-1) forward, 5'-AGCAGCAGGTGTCCCAAAGA-3' and reverse, 5'-GTG CTGAAGACCTTAGGGCAGA-3'; IFN $\gamma$ forward, 5'-CGG CACAGTCATTGAAAGCCTA-3' and reverse, 5'-GTTGCT GATGGCCTGATTGTC-3'; interferon-induced GTP-binding protein Mx1 (Mx1) forward, 5'-AATGTGGACATTGCTACC ACAGA-3' and reverse, 5'-GGTTCCGCATCACATCCA AG-3'; Viperin forward, 5'-CCGCAGTAACTCAGCTCA TGGA-3' and reverse, 5'-CTGAGCATTAGACCTCATCTG GACA-3'; 2'-5'-oligoadenylate synthase 1A (Oas1a) forward, 5'-TGCTGCCAGCCTTTGATGTC-3' and reverse, 5'-TCC TCGATGAGGATGGCATAGA-3'; ISG15 forward, 5'-CTG TGAGAGCAAGCAGCCAGA-3' and reverse, 5'-GAGTTA GTCACGGACACCAGGAA-3'.

In vivo virus quantitation. Methods for total RNA extraction and purification were performed as described above. RT-qPCR was performed using TaqMan Fast Virus 1-Step Master Mix (Thermo Fisher Scientific, Inc.) and a LightCycler 480 (Roche Diagnostics). The PCR conditions were as follows: 2-Step Cycling, $50^{\circ} \mathrm{C}$ for $5 \mathrm{~min}$ hold, 45 cycles of $95^{\circ} \mathrm{C}$ for $20 \mathrm{sec}$ and $60^{\circ} \mathrm{C}$ for $30 \mathrm{sec}$. All values were normalized to the expression of GAPDH and calculated using the $2^{-\Delta \Delta C q}$ method (32). The specific forward and reverse primer pairs and probes used are listed as follows: GAPDH forward, 5'-CTTCACCACCATGGAGAA GGC-3' and reverse, 5'-GGCATGGACTGTGGTCATGAG-3'; GAPDH probe, 5'-(6-FAM)-CCTGGCCAAGGTCATCCA TGACAACTTT-(TAMRA)-3'; DENV Eden2 forward, 5'-CAT ATTGACGCTGGGAAAGA-3' and reverse, 5'-AGAACCTGT TGATTCAAC-3'; DENV Eden2 probe, 5'-(6-FAM)-CTGTCT CCTCAGCATCATTCCAGGCA-(TAMRA)-3'.

Statistical analysis. The data of virus titer and NS1 ELISA were presented as the mean + the value of each individual. All other data were presented as the mean \pm standard error of the mean. Data from the in vivo study were analyzed using an unpaired Student's t-test. Data from the in vitro study were analyzed using one-way analysis of variance followed by Dunnett's test. All statistical analyses were performed using the Excel-Toukei 2012 software program (Social Survey Research Information, Tokyo, Japan). $\mathrm{P}<0.05$ was considered to indicate a statistically significant difference.

\section{Results}

Oral administration of LC-plasma inhibits DENV infection. To investigate whether the pDC-stimulative LAB, LC-plasma, is effective for the prevention of DENV infection in mice, the mouse-infectious DENV strain Eden2 was used. As DENV Eden 2 has been previously reported to infect the spleen and

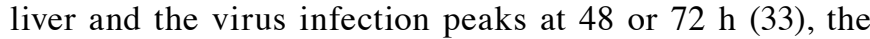
relative virus titers were assessed at 48 and $72 \mathrm{~h}$ following DENV infection to examine the effect of LC-plasma on DENV infection. The study design is presented in Fig. 1A. The mice were orally administered LC-plasma for two weeks, and subsequently were infected with a titer of $1 \times 10^{7}$ pfu of DENV Eden 2 by intraperitoneal injection. Oral intake of LC-plasma was continued for 2-3 days subsequent to DENV infection. A total of 2-3 days following DENV infection, 8 mice from each group were sacrificed, and the spleens, liver and blood were collected. No animals succumbed to DENV infection and any behavioral change was not observed. As presented in Fig. 1B, the relative virus titer in the spleens of the LC-plasma group $48 \mathrm{~h}$ subsequent to infection was significantly lower compared with that in the control group $(\mathrm{P}<0.01)$. On the other hand, there was no significant difference in the relative virus titers in the spleens at $72 \mathrm{~h}$ after infection between the two groups. The relative virus titer in the liver is presented in Fig. 1C. In the LC-plasma group, the relative virus titers at 48 and $72 \mathrm{~h}$ following infection were significantly lower compared with that of the control group $(\mathrm{P}=0.10$ and $\mathrm{P}=0.11$, respectively). NS1 antigen, which is a non-structural protein of the DENV (34), was also measured in the blood and the amount of NS1 at $48 \mathrm{~h}$ in the LC-plasma group was significantly lower compared with the control group $(\mathrm{P}<0.05)$. However, there was no difference in the amount of NS1 between the two groups $72 \mathrm{~h}$ after infection (Fig. 1D). These results suggested that oral administration of LC-plasma is effective in the prevention of DENV infection.

LC-plasma alleviates DENV-mediated inflammation. To evaluate the effect of LC-plasma on the suppression of inflammatory responses induced by DENV infection, the expression levels of inflammatory cytokine genes in the spleen and liver at 48 and $72 \mathrm{~h}$ subsequent to DENV infection were assessed by RT-qPCR. As presented in Fig. 2A, in the LC-plasma group, the expression levels of IL-6 $(\mathrm{P}<0.01)$, MCP-1 $(\mathrm{P}<0.05)$ and IFN- $\gamma(\mathrm{P}<0.05)$ in the spleen $72 \mathrm{~h}$ after infection were significantly lower compared with that in the control group. In addition, TNF- $\alpha$ expression levels in the spleen at 48 and $72 \mathrm{~h}$ after infection were non-significantly lower compared with that of the control group $(\mathrm{P}=0.08$ and $\mathrm{P}=0.08$, respectively). However, only TNF- $\alpha$ expression levels in the liver $48 \mathrm{~h}$ after infection were significantly lower compared with the control group $(\mathrm{P}<0.05)$, whereas the expression of other cytokine genes in the liver remained unchanged. These results suggest that the oral administration of LC-plasma alleviated inflammatory responses in DENV infected tissue.

Oral administration of $L C$-plasma enhances anti-viral factors in the spleen and CDIIc cells in the absence of the virus. It was demonstrated that oral intake of LC-plasma is effective against DENV infection and the associated inflammatory response. To elucidate how orally administered LC-plasma exerts an inhibitory effect on DENV, the expression of anti-viral factor genes in the spleen were evaluated, which is a primary site of DENV infection (33). Subsequent to two weeks of LC-plasma intake, 10 mice from each group were sacrificed, the spleens were harvested and the expression of anti-viral factor genes in the spleen were measured using RT-qPCR. 
A

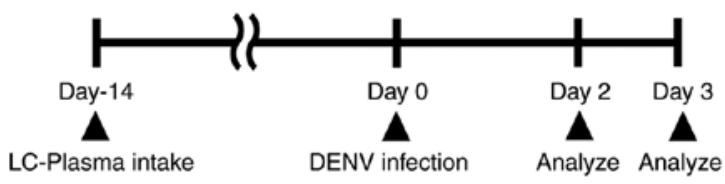

C

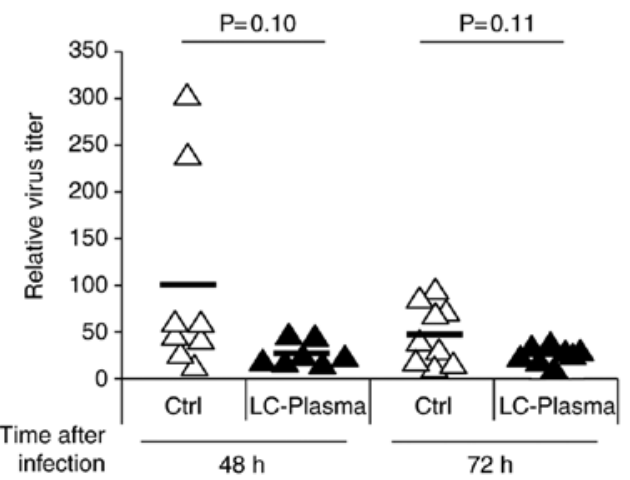

B
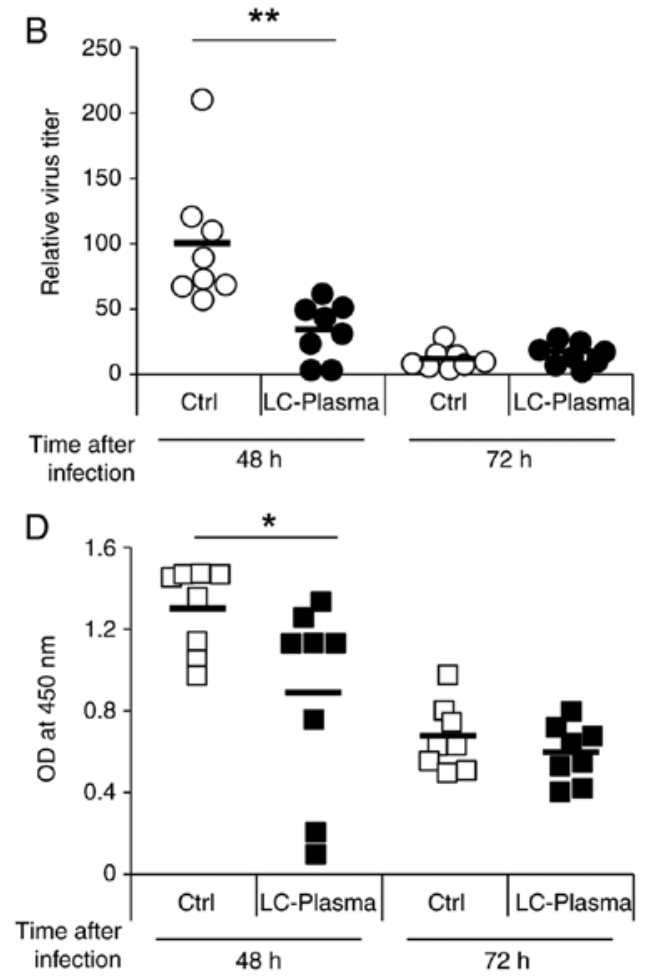

Figure 1. Effect of LC-plasma on DENV infection. (A) Experimental procedure for DENV infection. Mice in the control and LC-plasma groups were fed a diet with or without $1 \mathrm{mg} / \mathrm{head} / \mathrm{day}$ LC-plasma during the study period (day-14 to 3). Mice were intraperitoneally infected with DENV Eden2 at a titer of $1 \times 10^{7}$ pfu on day 0. A total of 2-3 days after DENV infection, a total of 8 mice were sacrificed from each group, and spleens, livers and blood were harvested. Total RNA was then extracted from spleen and liver. Relative virus titers in the (B) spleen and (C) liver were measured by reverse transcription-quantitative polymerase chain reaction normalized to GAPDH. (D) Quantity of NS1 peptide in blood measured using an enzyme-linked immunosorbent assay. Each dot corresponds to an individual mouse. The line indicates the mean concentration. ${ }^{*} \mathrm{P}<0.05$ and ${ }^{* * *} \mathrm{P}<0.01$ with comparisons shown by lines. LC-plasma, Lactococcus lactis strain plasma; DENV, dengue virus; Ctrl, control; OD, optical density.
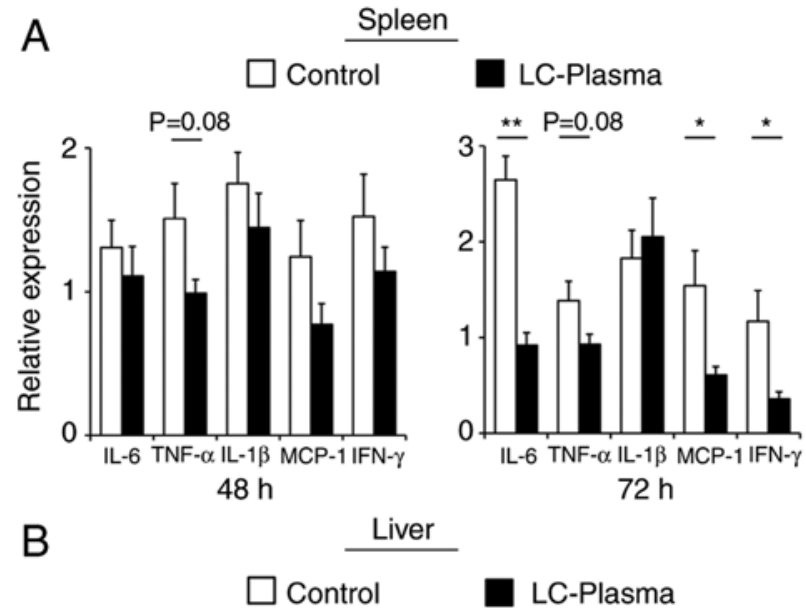

Liver

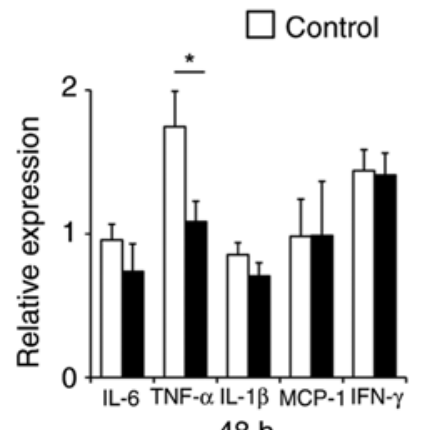

$48 \mathrm{~h}$

LC-Plasma

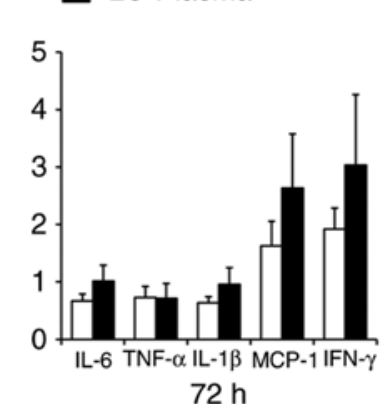

Figure 2. Effect of LC-plasma on DENV-mediated inflammation. Total RNA was extracted from the (A) spleen and (B) liver in each group. Expression levels of IL-6, TNF- $\alpha$, IL-1 $\beta$, MCP-1 and IFN- $\gamma$ genes were evaluated by reverse transcription-quantitative polymerase chain reaction normalized to GAPDH. Data are presented as the mean \pm standard error of the mean. ${ }^{*} \mathrm{P}<0.05$ and ${ }^{* *} \mathrm{P}<0.01$ with comparisons shown by lines. LC-plasma, Lactococcus lactis strain plasma; DENV, dengue virus; IL, interleukin; TNF- $\alpha$, tumor necrosis factor $\alpha$; MCP-1, monocyte chemoattractant protein 1; IFN- $\gamma$, interferon $\gamma$. 
A

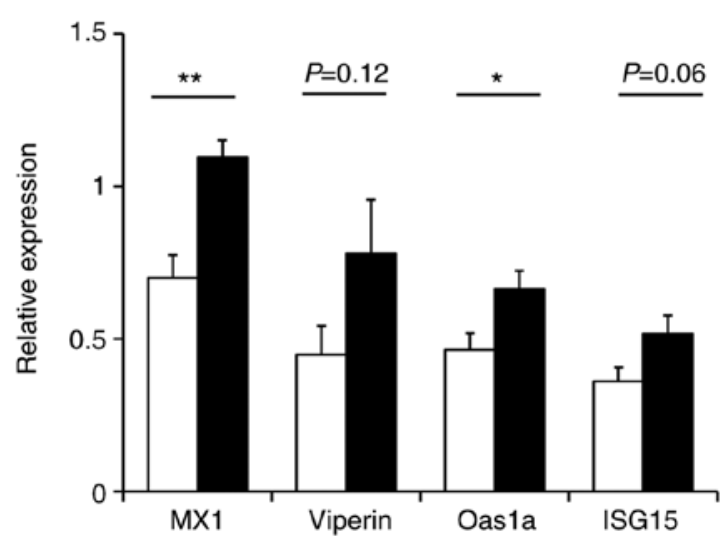

CD11c cells

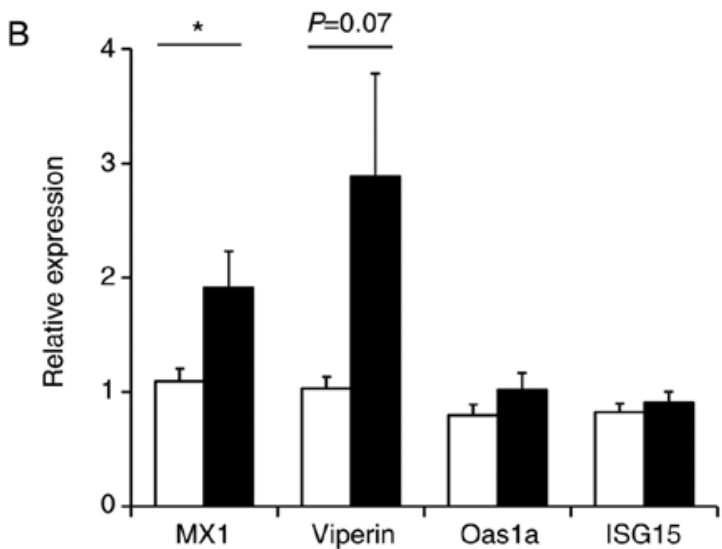

Figure 3. Effect of LC-plasma on anti-viral factor gene expression in spleen and $\mathrm{CD} 11 \mathrm{c}^{+}$cells derived from splenocytes. Mice in the control and LC-plasma groups ( $\mathrm{n}=10$ each) were fed a diet with or without $1 \mathrm{mg} / \mathrm{head} / \mathrm{day}$ LC-plasma for 2 weeks. A total of 2 weeks later, spleens were harvested from each mouse. Splenocytes were prepared and a portion of the splenocytes were collected for RNA extraction. $\mathrm{CD} 11 \mathrm{c}^{+}$cells were separated from the remaining splenocytes by magnetic separation and collected for RNA extraction. Total RNA was extracted from the (A) splenocytes and (B) CD11 $\mathrm{c}^{+}$ cells to analyze Mx1, Viperin, Oas1a and ISG15 expression levels by reverse transcription-quantitative polymerase chain reaction normalized to GAPDH. Data are presented as the mean \pm standard error of the mean. ${ }^{*} \mathrm{P}<0.05$ and ${ }^{* *} \mathrm{P}<0.01$ with comparisons shown by lines. LC-plasma, Lactococcus lactis strain plasma; Mx1, interferon-induced GTP-binding protein Mx1; Oas1a, 2'-5'-oligoadenylate synthase 1A; ISG15, IFN-stimulated gene 15.

As presented in Fig. 3A, oral administration of LC-plasma significantly enhanced the expression levels of $\mathrm{Mx} 1(\mathrm{P}<0.01)$ and Oasla $(\mathrm{P}<0.05)$ compared with the control group. In addition, LC-plasma non-significantly increased the expression levels of Viperin and ISG15 compared with the control group ( $\mathrm{P}=0.12$ and $\mathrm{P}=0.06$, respectively). Thus, LC-plasma administration caused the induction of anti-viral factor gene expression in the spleen. Among the various immune cells in the spleen, $\mathrm{CD} 11 \mathrm{c}^{+}$cells are the major target cells for DENV infection (33). Therefore, anti-viral gene expression in $\mathrm{CD}_{11 \mathrm{c}^{+}}$ cells was also measured, which were magnetically separated from the spleen tissue. As presented in Fig. 3B, LC-plasma administration significantly induced the expression levels of $\mathrm{Mx} 1(\mathrm{P}<0.05)$ and also non-significantly enhanced Viperin expression levels $(\mathrm{P}=0.07)$ in $\mathrm{CD} 1 \mathrm{c}^{+}$cells from the spleen compared with the control group. On the other hand, Oasla and
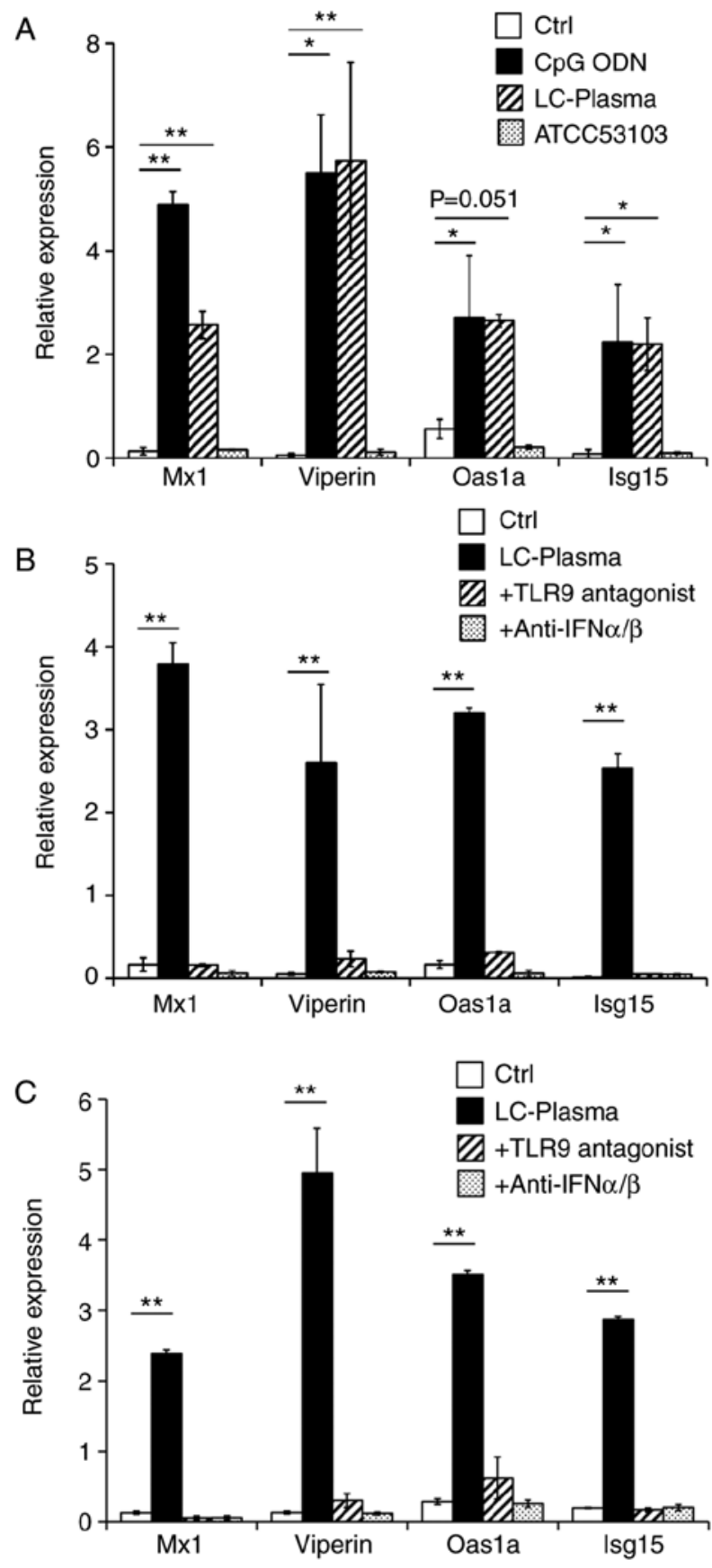

Figure 4. Enhancement of anti-viral factor gene expression mediated by LC-plasma via type I IFNs in vitro. (A) Flt-3L induced BMDC were cultured with CpG ODN, LC-plasma or ATCC 53103 for $24 \mathrm{~h}$. Subsequent to culturing, BMDC were collected for RNA extraction. (B) Flt-3L-induced BMDC were cultured with LC-plasma in the presence or absence of a TLR9 antagonist or anti-IFN- $\alpha / \beta$ antibody for $24 \mathrm{~h}$. After culturing, the supernatant was collected and another sample of Flt-3L-induced BMDC was cultured with the collected supernatant for $24 \mathrm{~h}$. Subsequent to culturing with the supernatant, BMDC were collected for RNA extraction. (C) Flt-3L-induced BMDC were cultured with LC-plasma in the presence or absence of TLR9 antagonist or anti-IFN- $\alpha / \beta$ antibody for $24 \mathrm{~h}$. Subsequent to culturing, the supernatant was collected and the splenocytes were cultured with the collected supernatant for $24 \mathrm{~h}$. After culturing with the supernatant, splenocytes were collected for RNA extraction. Total RNA was extracted from each cell culture to analyze Mx1, Viperin, Oas1a and ISG15 expression by reverse transcription-quantitative polymerase chain reaction normalized to GAPDH. Data are presented as the mean \pm standard error of the mean. ${ }^{*} \mathrm{P}<0.05$ and ${ }^{* *} \mathrm{P}<0.01$ with comparisons shown by lines. LC-plasma, Lactococcus lactis strain plasma; IFN, interferon; Flt-3L, FMS-like tyrosine kinase 3 ligand; BMDC, bone-marrow derived dendritic cells; ODN, oligodeoxyribonucleotides; TLR9, Toll-like receptor 9; Mx1, interferon-induced GTP-binding protein Mx1; Oas1a, 2'-5'-oligoadenylate synthase 1A; ISG15, IFN-stimulated gene 15 . 
ISG15 expression levels in CD11c cells were not influenced by LC-plasma administration. These results demonstrate that LC-plasma may alleviate DENV infection through an increase of anti-viral factor gene expression in infected tissue.

LC-plasma enhances anti-viral factors through type I INF in vitro. In vivo studies revealed that the oral treatment of LC-plasma enhanced the anti-viral factors in DENV infected tissue, which may be one mechanism used by LC-plasma to alleviate DENV infection. As LC-plasma strongly induces type I IFNs through pDC activation, it was investigated whether the effect of LC-plasma on the enhancement of anti-viral factors was dependent on type I IFNs through the use of in vitro studies. To assess this hypothesis, Flt-3L-induced bone-marrow derived DC (BMDC) were used, which includes pDC and $\mathrm{CDC}$ (35). BMDC were stimulated with the TLR9 agonist CpG ODN, or with the LAB strains of LC-plasma or Lactobacillus rhamnosus strain GG (ATCC 53103). After $24 \mathrm{~h}$ of stimulation, BMDC were collected and the expression levels of anti-viral genes were analyzed. As presented in Fig. 4A, stimulation of BMDC with CpG ODN or LC-plasma significantly increased the expression of the anti-viral factor genes compared with the control group $(\mathrm{P}<0.05$; Fig. $4 \mathrm{~A})$. In contrast, ATCC 53103 did not influence the expression of the anti-viral genes. To investigate whether this LC-plasma effect is dependent on soluble factors including interleukins or interferons produced by BMDC, the supernatants were collected and another sample of BMDC was re-stimulated with these supernatants. As presented in Fig. 4B, the supernatants from BMDC stimulated with LC-plasma also significantly enhanced anti-viral gene expression in another BMDC sample $(\mathrm{P}<0.01)$. However, this effect was completely abolished by TLR9 antagonist treatment. Furthermore, anti-IFN $\alpha / \beta$ antibody treatment with BMDC stimulated with LC-plasma also blocked this effect. To investigate whether this LC-plasma effect also occurs in the spleen, which is the major DENV infected tissue, supernatants collected from BMDC stimulated with LC-plasma were added to splenocytes. As presented in Fig. 4C, LC-plasma supernatants also significantly enhanced anti-viral gene expression levels compared with the control group $(\mathrm{P}<0.01)$ and this effect was completely abolished by TLR9 antagonist or anti-IFN $\alpha / \beta$ antibody treatment. Based on these results, it appears that LC-plasma may attenuate DENV infection through type I IFN production by pDCs.

\section{Discussion}

To date, despite major efforts, there exists no effective treatment or vaccine for DENV infection. It has been previously reported that the specific LAB, LC-plasma, stimulates pDCs to induce the production of a large quantity of type I IFNs (28). As pDCs and type I IFNs serve a pivotal role in anti-viral immune responses, it was predicted that $\mathrm{pDC}$-stimulative LAB would be effective against DENV. In the present study, the effect of LC-plasma on DENV infection was examined using a mouse model.

A major technical barrier in developing therapeutics or vaccines against DENV infection is the absence of a suitable animal model that mimics dengue disease. The first established animal models used immune-competent mice in which a high dose of DENV was administered intracranially (36). Other animal models included immunocompromised mice lacking type I and/or type II IFNs receptors $(37,38)$. However, neither of these models is suitable to evaluate treatment or vaccines for DENV as the route of infection is different from the usual DENV infection route in one model, and the other uses an immunocompromised mouse lacking the relevant immune receptor(s) (39). Previously, a DENV strain capable infecting mice, DENV Eden2, was reported $(33,40)$. This strain was used to infect wild-type mice by intraperitoneal injection and it was revealed be capable of replicating in the spleen and liver. These organs are also DENV-target organs in humans, so this strain is appropriate for evaluating the effectiveness of therapeutics for DENV. Therefore, the present study used the DENV Eden2 strain to evaluate the effect of LC-plasma on DENV infection in vivo, and revealed that LC-plasma is effective against DENV infection by measuring the virus titer in the spleen and the liver and the NS1 expression level in blood.

It was also revealed that inflammatory gene expression in DENV infected tissue was inhibited by oral administration of LC-plasma. DENV infection in children and adults usually results in dengue fever accompanied by a combination of symptoms that includes headache, retro-orbital pain, myalgia and hemorrhagic manifestations $(41,42)$. Certain patients including newborns and elderly people occasionally have an onset of dengue hemorrhagic fever, the severest form of dengue disease. Patients with dengue hemorrhagic fever manifest a cytokine storm, with high levels of circulating pro-inflammatory cytokines and chemokines, which results in endothelial damage and vascular leakage with hemorrhaging and shock $(6,42)$. Therefore, excessive inflammation induced by DENV infection results in severe dengue disease and the inhibition of inflammatory cytokines or chemokines may result in a reduction of DENV-associated inflammatory symptoms $(43,44)$. Thus, LC-plasma administration may contribute to the suppression of DENV pathogenesis.

A number of reports have demonstrated that type I IFNs serve a principal role in inhibiting DENV replication (12-15). One of the essential roles of type I IFNs is the induction of anti-viral factors, which are involved in the suppression of viral replication and release (16-18). Thus, it was predicted that LC-plasma administration may inhibit DENV replication through the enhancement of anti-viral factors in DENV infected tissues. To reveal the mechanism of the LC-plasma effect against DENV infection, it was investigated whether LC-plasma enhances the expression of anti-viral genes in DENV infected tissues and cells. The expression of four important anti-viral genes, Mx1, Viperin, Oas1a and Isg15, were assessed. Mx1 is an interferon-induced GTP-binding protein that prevents transcription of DENV by interacting with viral polymerase (45). Viperin is a multifunctional antiviral factor that inhibits the replication of DNA and RNA viruses including hepatitis $\mathrm{C}$ virus, cytomegalovirus, influenza virus and DENV (46). Isg15 is an ubiquitin-like interferon-stimulated protein that conjugates viral proteins, resulting in the suppression of viral release (47). Oas1a is a 2',5'-oligoadenylate synthase that synthesizes higher oligomers of 2'-5'-oligoadenylates from ATP. The oligoadenylates activate RNase L, which degrades viral RNAs (48). Oral administration of LC-plasma significantly enhanced Mx1 and 
Oas1a and non-significantly enhanced Viperin and Isg15 in the spleen. Furthermore, LC-plasma induced Mx1 and Viperin in CD11 $\mathrm{c}^{+}$cells, which is one of the cell types that DENV infects. The results of the present study indicate that LC-plasma may suppress DENV replication by inducing the expression of these anti-viral genes.

The in vitro experiments in the present study clearly demonstrated that LC-plasma increases the expression of anti-viral genes; and this effect was completely abolished by TLR9 antagonists or anti-type I IFN antibody treatment. However, another strain of LAB, ATCC 53103, which does not induce type I IFNs, did not have the ability to enhance anti-viral gene expression. These results demonstrate that pDC-stimulative LAB strongly inhibit viral infection through type I IFN production.

To date, various strains of LAB have been reported to have immune-modulatory effects by affecting immune cells, resulting in anti-pathogenic effects or improving allergy symptoms (49-51). In the present study, it was revealed for the first time that the pDC-stimulating LAB, LC-plasma, is effective against DENV infection. As LC-plasma also has this effect on different types of viruses $(29,30)$, pDC-stimulating LAB may additionally be effective against other viruses.

\section{Acknowledgements}

The authors would like to thank Dr. Ashley L. St. John in Duke-NUS Medical School (College Road, Singapore) for providing Dengue virus strain Eden 2 and Dr. Yo-ichi Suzuki in Osaka Medical College (Osaka, Japan) for providing C6/36 cells and Baby hamster kidney 21 cells.

\section{Funding}

No funding was received.

\section{Availability of data and materials}

The datasets used and/or analyzed during the current study are available from the corresponding author on reasonable request.

\section{Authors' contributions}

HS and NoY designed the experiments. HS, RT, MS and NoY performed the experiments and analyzed the data. NaY, NoY and OK supervised the experiments. HS wrote the paper and all authors read and approved the final manuscript.

\section{Ethics approval and consent to participate}

All animal experiments were performed in strict accordance with the guidelines for the care and use of laboratory animals of Juntendo University (Tokyo, Japan) and Kirin Company (Kanagawa, Japan). The studies were approved by the Committee for Animal Experimentation of Juntendo University and Kirin Company.

\section{Patient consent for publication}

Not applicable.

\section{Competing interests}

The authors declare that they have no competing interests.

\section{References}

1. Diamond MS and Pierson TC: Molecular Insight into dengue virus pathogenesis and its implications for disease control. Cell 162: 488-492, 2015.

2. Holmes EC and Burch SS: The causes and consequences of genetic variation in dengue virus. Trends Microbiol 8: 74-77, 2000.

3. Halstead SB: Dengue. Lancet 370: 1644-1652, 2007.

4. Gubler DJ: Dengue/dengue haemorrhagic fever: History and current status. Novartis Found Symp 277: 3-16; 16-22, 71-73, 251-253, 2006.

5. Srikiatkhachorn A, Mathew A and Rothman AL: Immunemediated cytokine storm and its role in severe dengue. Semin Immunopath 39: 563-574, 2017.

6. Guzman MG and Kouri G: Dengue: An update. Lancet Infect Dis 2: 33-42, 2002.

7. Kalayanarooj S, Vaughn DW, Nimmannitya S, Green S, Suntayakorn S, Kunentrasai N, Viramitrachai W, Ratanachu-eke S, Kiatpolpoj S, Innis BL, et al: Early clinical and laboratory indicators of acute dengue illness. J Infect Dis 176: 313-321, 1997.

8. Fagundes CT, Costa VV, Cisalpino D, Souza DG and Teixeira MM: Therapeutic opportunities in dengue infection. Drug Devel Res 72: 480-500, 2011.

9. Perry AK, Chen G, Zheng D, Tang $\mathrm{H}$ and Cheng G: The host type I interferon response to viral and bacterial infections. Cell Res 15: 407-422, 2005.

10. Stetson DB and Medzhitov R: Type I interferons in host defense. Immunity 25: 373-381, 2006.

11. Fleming SB: Viral inhibition of the IFN-induced JAK/STAT signalling pathway: Development of live attenuated vaccines by mutation of viral-encoded IFN-antagonists. Vaccines 4: pii: E23, 2016.

12. Schoggins JW, Dorner M, Feulner M, Imanaka N, Murphy MY, Ploss A and Rice CM: Dengue reporter viruses reveal viral dynamics in interferon receptor-deficient mice and sensitivity to interferon effectors in vitro. Proc Natl Acad Sci USA 109: 14610-14615, 2012.

13. Zellweger RM, Prestwood TR and Shresta S: Enhanced infection of liver sinusoidal endothelial cells in a mouse model of antibody-induced severe dengue disease. Cell Host Microbe 7: 128-139, 2010.

14. Zust R, Toh YX, Valdes I, Cerny D, Heinrich J, Hermida L, Marcos E, Guillén G, Kalinke U, Shi PY, et al: Type I interferon signals in macrophages and dendritic cells control dengue virus infection: Implications for a new mouse model to test dengue vaccines. J Virol 88: 7276-7285, 2014.

15. Sarathy VV, White M, Li L, Gorder SR, Pyles RB, Campbell GA, Milligan GN, Bourne N and Barrett AD: A lethal murine infection model for dengue virus 3 in AG129 mice deficient in type I and II interferon receptors leads to systemic disease. J Virol 89: 1254-1266, 2015.

16. Diamond MS and Harris E: Interferon inhibits dengue virus infection by preventing translation of viral RNA through a PKR-independent mechanism. Virology 289: 297-311, 2001.

17. Helbig KJ, Carr JM, Calvert JK, Wati S, Clarke JN, Eyre NS, Narayana SK, Fiches GN, McCartney EM and Beard MR: Viperin is induced following dengue virus type-2 (DENV-2) infection and has anti-viral actions requiring the C-terminal end of viperin. PLoS Negl Trop Dis 7: e2178, 2013.

18. Hishiki T, Han Q, Arimoto K, Shimotohno K, Igarashi T, Vasudevan SG, Suzuki Y and Yamamoto N: Interferon-mediated ISG15 conjugation restricts dengue virus 2 replication. Biochem Biophys Res Commun 448: 95-100, 2014.

19. Siegal FP, Kadowaki N, Shodell M, Fitzgerald-Bocarsly PA, Shah K, Ho S, Antonenko S and Liu YJ: The nature of the principal type 1 interferon-producing cells in human blood. Science 284: 1835-1837, 1999.

20. Cella M, Jarrossay D, Facchetti F, Alebardi O, Nakajima H, Lanzavecchia A and Colonna M: Plasmacytoid monocytes migrate to inflamed lymph nodes and produce large amounts of type I interferon. Nat Med 5: 919-923, 1999.

21. Lund JM, Alexopoulou L, Sato A, Karow M, Adams NC, Gale NW, Iwasaki A and Flavell RA: Recognition of single-stranded RNA viruses by Toll-like receptor 7. Proc Natl Acad Sci USA 101: 5598-5603, 2004. 
22. Hemmi H, Takeuchi O, Kawai T, Kaisho T, Sato S, Sanjo H, Matsumoto M, Hoshino K, Wagner H, Takeda K, et al: A Toll-like receptor recognizes bacterial DNA. Nature 408: 740-745, 2000.

23. Bauer S, Kirschning CJ, Häcker H, Redecke V, Hausmann S, Akira S, Wagner H and Lipford GB: Human TLR9 confers responsiveness to bacterial DNA via species-specific CpG motif recognition. Proc Natl Acad Sci USA 98: 9237-9242, 2001.

24. Gilliet M, Cao W and Liu YJ: Plasmacytoid dendritic cells: Sensing nucleic acids in viral infection and autoimmune diseases. Nat Rev Immunol 8: 594-606, 2008.

25. Perdigon G, Fuller R and Raya R: Lactic acid bacteria and their effect on the immune system. Current Issues Intest Microbiol 2: 27-42, 2001

26. Parcina M, Wendt C, Goetz F, Zawatzky R, Zähringer U, Heeg K and Bekeredjian-Ding I: Staphylococcus aureus-induced plasmacytoid dendritic cell activation is based on an IgG-mediated memory response. J Immunol 181: 3823-3833, 2008.

27. Piccioli D, Sammicheli C, Tavarini S, Nuti S, Frigimelica E, Manetti AG, Nuccitelli A, Aprea S, Valentini S, Borgogni E, et al: Human plasmacytoid dendritic cells are unresponsive to bacterial stimulation and require a novel type of cooperation with myeloid dendritic cells for maturation. Blood 113: 4232-4239, 2009.

28. Jounai K, Ikado K, Sugimura T, Ano Y, Braun J and Fujiwara D: Spherical lactic acid bacteria activate plasmacytoid dendritic cells immunomodulatory function via TLR9-dependent crosstalk with myeloid dendritic cells. PLoS One 7: e32588, 2012.

29. Jounai K, Sugimura T, Ohshio K and Fujiwara D: Oral administration of Lactococcus lactis subsp. lactis JCM5805 enhances lung immune response resulting in protection from murine parainfluenza virus infection. PLoS One 10: e0119055, 2015.

30. Jounai K, Sugimura T, Morita Y, Ohshio K and Fujiwara D: Administration of Lactococcus lactis strain Plasma induces maturation of plasmacytoid dendritic cells and protection from rotavirus infection in suckling mice. Int Immunopharmacol 56: 205-211, 2018

31. Castillo Ramirez JA and Urcuqui-Inchima S: Dengue virus control of type I IFN responses: A history of manipulation and control. J Interferon Cytokine Res 35: 421-430, 2015.

32. Livak KJ and Schmittgen TD: Analysis of relative gene expression data using real-time quantitative PCR and the $2^{-\Delta \Delta C}$ method. Methods 25: 402-408, 2001.

33. St John AL, Rathore AP, Raghavan B, Ng ML and Abraham SN: Contributions of mast cells and vasoactive products, leukotrienes and chymase, to dengue virus-induced vascular leakage. Elife 2 : e00481, 2013.

34. da Costa VG, Marques-Silva AC and Moreli ML: A meta-analysis of the diagnostic accuracy of two commercial NS1 antigen ELISA tests for early dengue virus detection. PLoS One 9: e94655, 2014.

35. Brawand P, Fitzpatrick DR, Greenfield BW, Brasel K, Maliszewski CR and De Smedt T: Murine plasmacytoid pre-dendritic cells generated from Flt3 ligand-supplemented bone marrow cultures are immature APCs. J Immunol 169: $6711-6719,2002$
36. Raut CG, Deolankar RP, Kolhapure RM and Goverdhan MK Susceptibility of laboratory-bred rodents to the experimental infection with dengue virus type 2. Acta Virol 40: 143-146, 1996.

37. Johnson AJ and Roehrig JT: New mouse model for dengue virus vaccine testing. J Virol 73: 783-786, 1999.

38. Orozco S, Schmid MA, Parameswaran P, Lachica R, Henn MR, Beatty R and Harris E: Characterization of a model of lethal dengue virus 2 infection in C57BL/6 mice deficient in the alpha/beta interferon receptor. J Gen Virol 93: 2152-2157, 2012.

39. Zellweger RM and Shresta S: Mouse models to study dengue virus immunology and pathogenesis. Front Immunol 5: 151, 2014.

40. Syenina A, Jagaraj CJ, Aman SA, Sridharan A and St John AL: Dengue vascular leakage is augmented by mast cell degranulation mediated by immunoglobulin $\mathrm{Fc} \gamma$ receptors. Elife 4: doi: 10.7554/eLife.05291, 2015 .

41. Gubler DJ: Dengue and dengue hemorrhagic fever. Clin Microbiol Rev 11: 480-496, 1998.

42. Green S and Rothman A: Immunopathological mechanisms in dengue and dengue hemorrhagic fever. Curr Opin Infect Dis 19: 429-436, 2006

43. Srikiatkhachorn A: Plasma leakage in dengue haemorrhagic fever. Thromb Haemost 102: 1042-1049, 2009.

44. Chaturvedi UC, Agarwal R, Elbishbishi EA and Mustafa AS: Cytokine cascade in dengue hemorrhagic fever: Implications for pathogenesis. FEMS Immunol Med Microbiol 28: 183-188, 2000

45. Verhelst J, Hulpiau P and Saelens X: Mx proteins: Antiviral gatekeepers that restrain the uninvited. Microbiol Mol Biol Rev 77: 551-566, 2013.

46. Fitzgerald KA: The interferon inducible gene: Viperin. J Interferon Cytokine Res 31: 131-135, 2011.

47. Zhao C, Collins MN, Hsiang TY and Krug RM: Interferoninduced ISG15 pathway: An ongoing virus-host battle. Trends Microbiol 21: 181-186, 2013.

48. Elkhateeb E, Tag-El-Din-Hassan HT, Sasaki N, Torigoe D, Morimatsu M and Agui T: The role of mouse 2',5'-oligoadenylate synthetase 1 paralogs. Infect Genet Evol 45: 393-401, 2016.

49. Takeda S, Takeshita M, Kikuchi Y, Dashnyam B, Kawahara S, Yoshida H, Watanabe W, Muguruma M and Kurokawa M: Efficacy of oral administration of heat-killed probiotics from Mongolian dairy products against influenza infection in mice: Alleviation of influenza infection by its immunomodulatory activity through intestinal immunity. Int Immunopharmacol 11: 1976-1983, 2011.

50. Fujiwara D, Inoue S, Wakabayashi H and Fujii T: The anti-allergic effects of lactic acid bacteria are strain dependent and mediated by effects on both Th1/Th2 cytokine expression and balance. Int Arch Allergy Immunol 135: 205-215, 2004.

51. Wakabayashi H, Nariai C, Takemura F, Nakao W and Fujiwara D: Dietary supplementation with lactic acid bacteria attenuates the development of atopic-dermatitis-like skin lesions in $\mathrm{NC} / \mathrm{Nga}$ mice in a strain-dependent manner. Int Arch Allergy Immunol 145: 141-151, 2008. 